\title{
Primary Peritoneal Serous Papillary Adenocarcinoma
}

National Cancer Institute

\section{Source}

National Cancer Institute. Primary Peritoneal Serous Papillary Adenocarcinoma. NCI

Thesaurus. Code C7695.

A rare, serous papillary adenocarcinoma that arises from the lining of the peritoneum. It affects females. The clinical behavior and pathologic characteristics are similar to the serous papillary adenocarcinoma that arises from the ovary. 